\title{
Automatic Scan Planning for Magnetic Resonance Imaging of the Knee Joint
}

\author{
Stefan Bauer, ${ }^{1}$ Lucas E. Ritacco, ${ }^{2}$ Chris Boesch, ${ }^{3}$ Lutz-P. Nolte, ${ }^{1}$ and Mauricio Reyes ${ }^{1}$ \\ ${ }^{1}$ Institute for Surgical Technology and Biomechanics, University of Bern, Stauffacherstr. 78, 3014 Bern, Switzerland; ${ }^{2}$ Virtual \\ Planning and Navigation Unit, Hospital Italiano de Buenos Aires, Buenos Aires, Argentina; and ${ }^{3}$ Departments of Clinical \\ Research and Radiology, University of Bern, Bern, Switzerland
}

(Received 22 December 2011; accepted 15 March 2012)

Associate Editor Jeffrey L. Duerk oversaw the review of this article.

\begin{abstract}
Automatic scan planning for magnetic resonance imaging of the knee aims at defining an oriented bounding box around the knee joint from sparse scout images in order to choose the optimal field of view for the diagnostic images and limit acquisition time. We propose a fast and fully automatic method to perform this task based on the standard clinical scout imaging protocol. The method is based on sequential Chamfer matching of $2 \mathrm{D}$ scout feature images with a three-dimensional mean model of femur and tibia. Subsequently, the joint plane separating femur and tibia, which contains both menisci, can be automatically detected using an information-augmented active shape model on the diagnostic images. This can assist the clinicians in quickly defining slices with standardized and reproducible orientation, thus increasing diagnostic accuracy and also comparability of serial examinations. The method has been evaluated on 42 knee MR images. It has the potential to be incorporated into existing systems because it does not change the current acquisition protocol.
\end{abstract}

Keywords-Bounding box detection, Automatic alignment, Chamfer matching, Active shape model segmentation, Anatomy localization, Scout imaging.

\section{INTRODUCTION}

Magnetic resonance imaging (MRI) of the knee is becoming more important in daily clinical practice because it offers the possibility not only to diagnose bone fractures but also to draw conclusions about injuries of soft tissues such as cartilages, ligaments or the menisci. ${ }^{17}$ Following scout images (also called

Address correspondence to Stefan Bauer, Institute for Surgical Technology and Biomechanics, University of Bern, Stauffacherstr. 78,3014 Bern, Switzerland. Electronic mail: stefan.bauer@istb. unibe.ch "localizer" or "locator" images), multiple diagnostic series are acquired. The quality of these scout images is comparatively low and not sufficient for diagnosis, but they can be acquired in a very short time and they define anatomical structures sufficiently to prescribe the following sequences. As a standard procedure in a clinical environment, few slices (usually 3 slices) of $2 \mathrm{D}$ scout images, with sparse resolution in three orientations (axial, coronal, sagittal) are taken first, in order to determine the optimal field of view and slice orientation for the more detailed diagnostic images.

For a thorough analysis of the knee joint, the diagnostic image must contain left and right meniscus as well as anterior cruciate ligament (ACL) and posterior cruciate ligament (PCL), which means the orientation of the bounding box for image acquisition should also be taken into account. Currently, in clinical practice, the locator images are analyzed manually by a technician who decides about the field of view for the diagnostic images. This decision is based on alignment in parallel to the line interconnecting the lateral and medial condyles and in parallel to the tibia plateau for the menisci. An automatic method, which is seamlessly integrated into the clinical workflow could bring down both scan time and costs thanks to less user interaction. Furthermore, using an automatic approach, the selection of the scan field could be standardized and would be less variable because it does not depend on the skills and experience of the technician. In order to automate and facilitate the task, it would be desirable to have a software tool, which analyzes the locator images automatically in order to find an oriented bounding box for the field of view of diagnostic images. As a requirement, this tool has to be fast, robust with respect to the imaging protocol and should not include any user interaction. 


\section{Related Work}

Automatic scan planning (ASP) for magnetic resonance imaging has been applied to other anatomical structures before, e.g., brain, ${ }^{10}$ heart ${ }^{11}$ or pelvis. ${ }^{16}$ However, ASP for clinical workflow integration in knee MRI is a rather new topic and to the best of our knowledge, there are only five publications in this field so far. Bystrov et al. ${ }^{4}$ derive anatomical landmarks by adapting an active shape model to a $3 \mathrm{D}$ scout scan and use the obtained landmarks for defining the field of view. This method has been clinically evaluated by Lecouvet et al. ${ }^{15}$ Jolly et $a .^{12}$ took a different approach. They did not apply a model-based technique, but employ a combination of hidden Markov models and random walker algorithm for segmentation and condyle detection on 3D scout images instead. Finally, Zhan et al. ${ }^{19}$ suggest a completely different method based on a hierarchical redundant anatomy detection framework. This work has also been described in more detail and further evaluated in Zhan et $a{ }^{18}$ However, all the three different methods presented so far have in common that they require a new and modified fully 3D scout imaging protocol for alignment, whereas the current manual alignment is based on 2D scout slices. The 3D scout image is not introduced in the clinics yet.

In a related field for automatic scan prescription, Blumenfeld et al. ${ }^{1}$ and also Goldenstein et al. ${ }^{7}$ propose to use image registration techniques for automatic alignment. However, these methods are only intended to be employed for follow-up studies, and not for scan planning in general. They perform alignment on the diagnostic images, not on the scout images, and their processing times are beyond what is acceptable for standard scan planning in the clinics.

We developed a method for automatic scan planning of knee MRI exams, which is fast and can be seamlessly integrated into the clinical workflow. In contrast to all other methods for ASP presented so far, it can operate on the standard 2D locator images currently used in the clinics, and it does not require a new protocol for acquisition of isotropic 3D locator images. As an extension, we present a method to automatically define the oblique plane showing both menisci from the diagnostic images. This can help the clinician to quickly navigate to the structure of interest in large image datasets.

\section{MATERIALS AND METHODS}

We use a model-based approach with Chamfer matching on the currently employed standard 2D scout images for initial alignment and definition of the oriented bounding box (OBB). After the diagnostic images have been acquired accordingly, the joint plane separating femur and tibia can be detected using an informationaugmented active shape modeling (ASM) method. The workflow is illustrated in Fig. 1. The method has been implemented on a stand-alone system using the Insight Toolkit for Segmentation and Registration (ITK) ${ }^{9}$ and
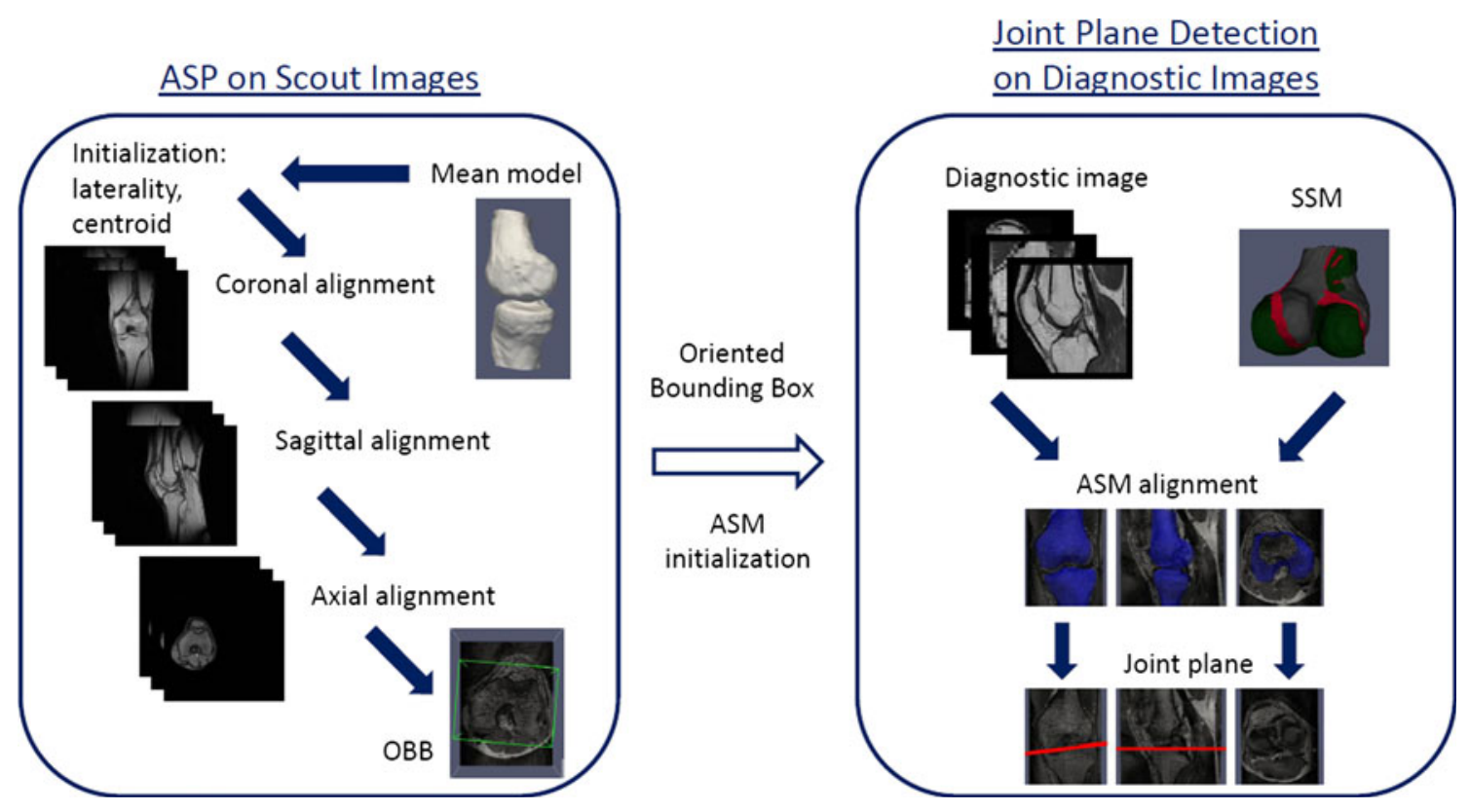

FIGURE 1. Illustration of the workflow: automatic scan planning is performed on the scout images in coronal, sagittal and axial view sequentially to obtain an oriented bounding box for acquisition of the high-quality diagnostic images. The outcome of this procedure also serves as an initialization for the active shape model alignment on the diagnostic images, from which the joint plane can be defined. 
the Visualization Toolkit (VTK). ${ }^{13}$ Both software libraries are open-source and based on $\mathrm{C}++$ with an open licensing model, which offers the possibility to integrate them into vendor systems. The method and workflow is described in the next sections in more detail.

\section{Definition of the Oriented Bounding Box}

The oriented bounding box defining the relevant region of the knee joint, is computed based on a Chamfer matching approach. ${ }^{2}$ An example of the 2D scout images on which it is applied, is shown in coronal, sagittal and axial acquisition view in Fig. 2.

Chamfer matching is a fast algorithm for alignment of a template model with a feature image. This is achieved by convolution of the template model with the image and a search for the best possible match. As a template for matching, we use a mean model of femur and tibia, generated from 190 manually segmented CT images from normal volunteers (data from Kozic et al. ${ }^{14}$ and Bou Sleiman et al. ${ }^{3}$ ). In our case, the feature image consists of the edges of the original image, which are obtained using a Canny edge detector. ${ }^{5}$ This method detects edges in an image based on the maxima of the gradient magnitude of the Gaussian-smoothed image and hysteresis thresholding. From the edge image, a Chamfer distance map is computed, which encodes the distance of every voxel in the image to the closest edge. The best match between the image and the template model can then be found by sliding the
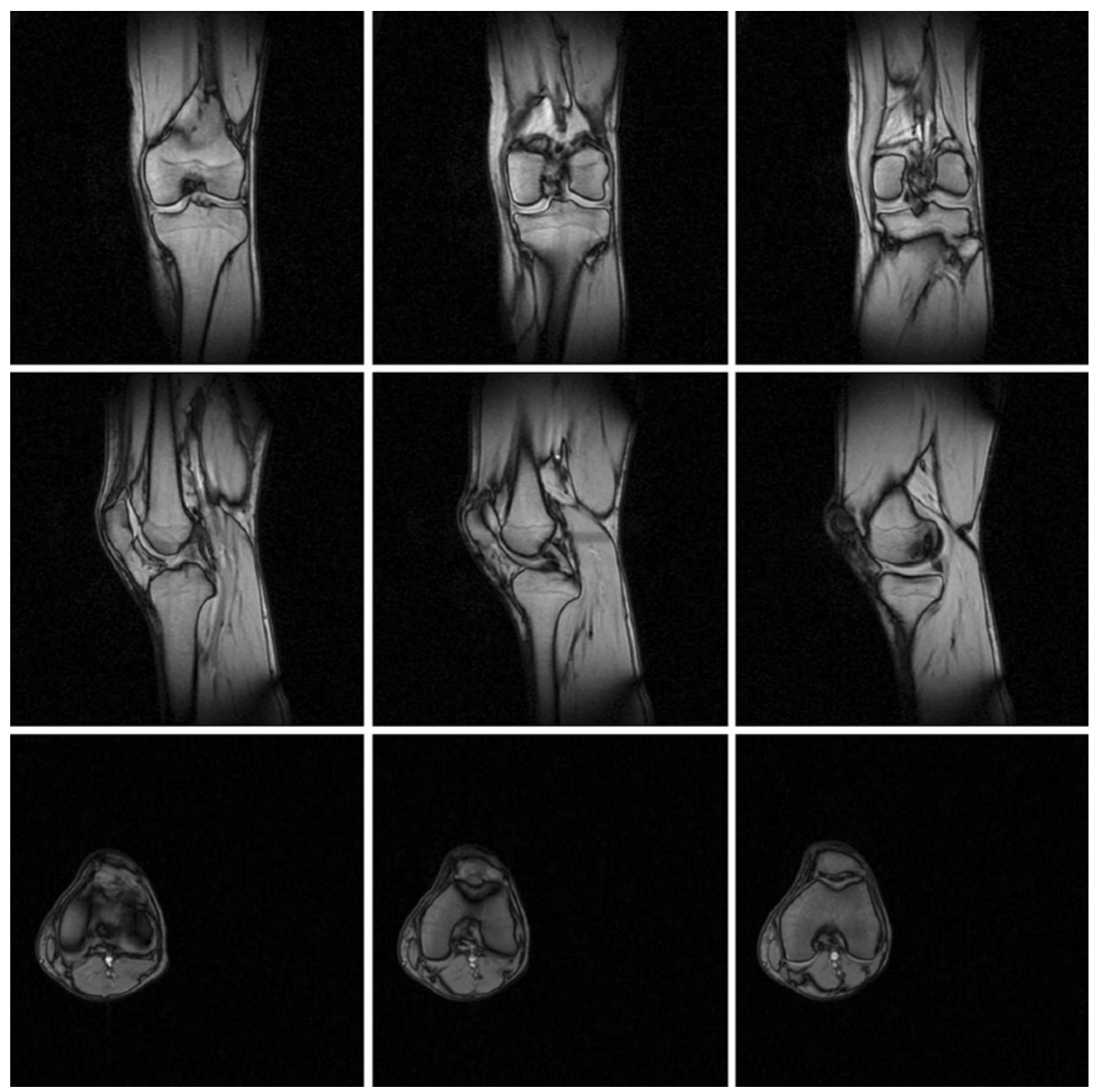

FIGURE 2. One example for the scout images (2D gradient echo sequence, $T_{R}=7.7 \mathrm{~ms}$ and $T_{E}=3.67 \mathrm{~ms}$ ), on which the Chamfer matching for $O B B$ definition is performed. Top row: the three slices in coronal acquisition. Center row: the three slices in sagittal acquisition. Bottom row: the three slices in axial acquisition. (9 $\mathrm{mm}$ slice spacing in each case). 
template over the distance map, calculating the Chamfer distance $d_{\text {chamfer }}$ at each step and choosing the position with minimum distance. Using a distance map instead of using the feature image directly speeds up the process significantly because the distance map can serve as a lookup table. The Chamfer distance $d_{\text {chamfer }}$ between the aligned model and the image is computed according to Eq. (1), where $\mathbf{M}$ corresponds to the mean model, $\mathbf{m}$ are the individual points of the model and $\mathbf{I}$ corresponds to the distance map.

$$
d_{\text {chamfer }}(\mathbf{M}, \mathbf{I})=\frac{1}{|\mathbf{M}|} \sum_{\mathbf{m} \in \mathbf{M}} d_{\mathbf{I}}(\mathbf{m})
$$

So, for each point $\mathbf{m}$ of the model $\mathbf{M}$, the distance $d_{\mathbf{I}}(\mathbf{m})$ to the closest feature point in the image is computed using the distance map I. Taking the mean over the number of all points of the model $|\mathbf{M}|$, we get the average distance to the nearest edge. By sliding the model over the distance map in different scales and with different translations and rotations, the expression for $d_{\text {chamfer }}(\mathbf{M}, \mathbf{I})$ in Eq. (1) should be minimized.

In our implementation, an initial alignment is based on matching the centroids of the feature image and the model. Next, a laterality detection determines if the left or right knee is being imaged. This is based on the location of the feature image centroid. Then a refinement cascade for model alignment using the previously described Chamfer matching process is started, which operates on the coronally, sagittally and axially acquired images sequentially. This is according to the standard acquisition order of the clinical protocol. The process allows for a pipeline operation, where the previous view can be processed while the next view is already being acquired. First, we operate on the coronal scout images, where we perform Chamfer matching with the mean model at three different scales and translations in $x, y, z$-direction. The alignment with minimum Chamfer distance on the coronal images serves as initialization for a more refined alignment on sagittal scout images, where translations in $x, y, z$-direction in a smaller range are allowed. Finally, on the axial scout images we allow for some more small translations in $x$ - and $y$-direction, before a rotation around the $z$-axis is performed. The alignment of the model with minimum Chamfer distance $d_{\text {chamfer }}$ after scaling, translations and rotations is chosen as the best alignment. Based on the aligned model, the oriented bounding box for acquisition of the diagnostic images can be determined. The extremal points of the aligned model define the corners of the bounding box and two distinguished points on both condyles of the aligned model define the rotation angle. A safety margin of $6 \mathrm{~mm}$ is added to the bounding box in $x$ - and $y$-direction in order to be able to handle small misalignments without risking an incomplete coverage of the knee joint.

\section{Definition of the Joint Plane}

After obtaining the diagnostic image, the joint plane separating femur and tibia can be found using a method based on active shape modeling (ASM). ${ }^{6,8}$ An example of a diagnostic image, on which the algorithm operates is depicted in Fig. 3.

Active shape modeling uses statistical shape models $(\mathrm{SSM})^{6}$ of the structure of interest and adapts them to the same structure in the patient. We build the shape model from the same 190 manually segmented CT images of femurs and tibia employed before and we use the mean shape together with its first ten modes of variation computed by Principal Component Analysis (PCA) (more than $90 \%$ of variation retained). The information contained in the shape model is augmented by defining landmark points on the model, which can deform along with the model during adaptation to the patient image. The landmarks were defined together with a clinical expert on both femoral condyles and on the tibia plateau. The ASM search is
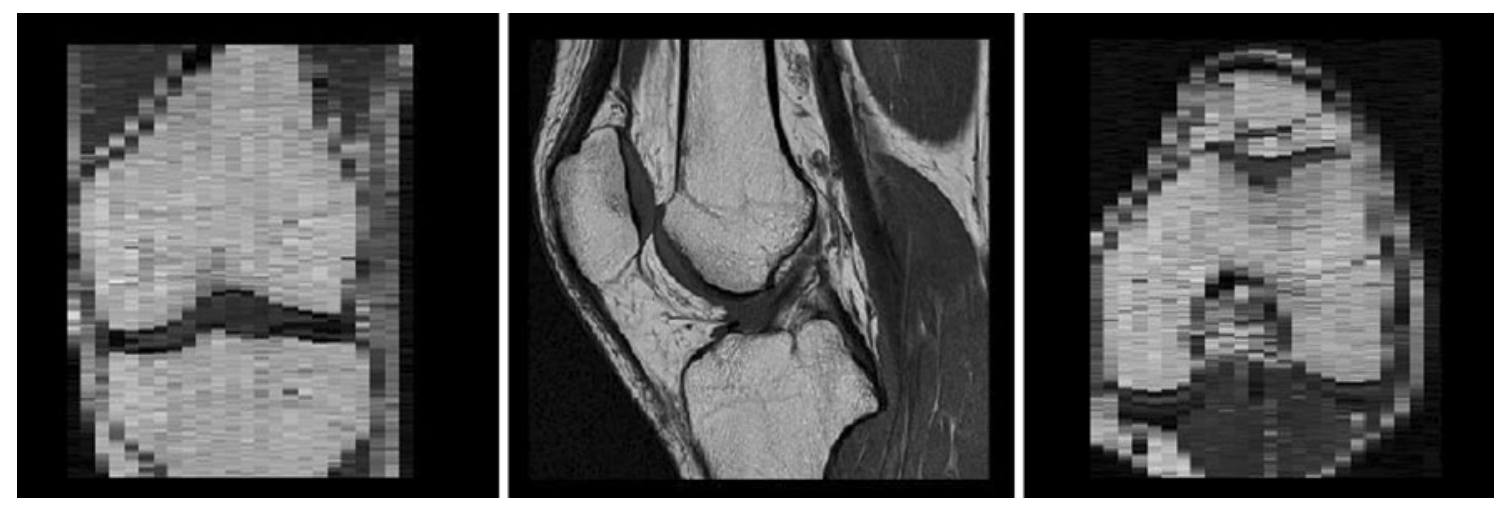

FIGURE 3. One example of a diagnostic image (2D spin echo sequence, $T_{\mathrm{R}}=550 \mathrm{~ms}$ and $T_{\mathrm{E}}=16 \mathrm{~ms}$ ), in sagittal acquisition order, on which the ASM algorithm is applied for defining the joint plane. From left to right: coronal view, sagittal view, axial view.

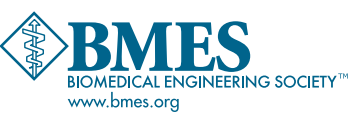


initialized using the laterality and coordinates for femur and tibia computed by the initial Chamfer matching process. We use two separate SSMs for femur and tibia, whereas the femur alignment is performed first and the tibia alignment second.

Active shape model alignment ${ }^{6,8}$ is a shape-constrained segmentation method, which requires a sufficiently good pre-alignment. After pre-alignment based on the outcome of the Chamfer matching process, a search along the normals of the initially aligned model for the most probable structure of interest in the image is performed. In this case, the structure of interest is found based on the gradient-magnitude filtered diagnostic image, in which we look for the largest gradient along the normals of each point in the model. This is supposed to correspond to the edge of the bone. In order to increase robustness, we set an additional tissue constraint, which ensures that the normalized image intensities inside the model correspond to actual bone intensities. After having found tentative points of the structure of interest, the model is adapted toward these points in an iterative process. This is done using a similarity transformation (7 degrees of freedom including scaling, translations and rotations ${ }^{20}$ ) and local deformations (driven by the PCA modes of the $\mathrm{SSM}^{6}$ ) to iteratively align and deform the model to the structure of interest. The process is stopped once the changes in alignment are small or the maximum number of iterations has been reached. Mathematically, this iterative alignment is formulated as a minimization problem with the objective to find the parameters of the transformation matrix $\mathbf{T}$ and the PCA-based deformation parameters $\mathbf{b}$, which minimize the error measure $e$ between the points of the model and the feature points.

$$
e=\underset{\mathbf{T}, \mathbf{b}}{\arg \min }\left\{\left\|\mathbf{y}-\mathbf{T}_{\mathbf{x}_{\mathrm{t}}, \mathrm{s}, \theta}(\overline{\mathbf{x}}+\mathbf{P b})\right\|\right\}
$$

In Eq. (2), $\mathbf{y}$ are the tentative feature points, which have been found in the image while searching for the strongest gradient along the normals of the model, $\mathbf{T}_{\mathbf{x}_{t}, \mathrm{~s}, \theta}$ is the transformation matrix for a similarity transform (with translation $\mathbf{x}_{\mathrm{t}}$, scaling $\mathrm{s}$ and rotation $\theta$ ), $\overline{\mathbf{x}}$ is the mean shape of the model, $\mathbf{P}$ is the matrix of eigenvectors of the model originating from the PCA, and $\mathbf{b}$ is a vector of deformation coefficients, which have to be determined. ${ }^{6}$ These coefficients are constrained to lie within 3 standard deviations from the respective eigenvalues in order to allow for plausible shapes only.

We use a multi-resolution approach ${ }^{8}$ with three resolution levels. This employs a coarse-to-fine strategy for model alignment on subsampled versions of the original image in order to increase robustness and speed of the algorithm. In the first resolution level, an approximate alignment with long search range along the normals of the model is done, which is further refined in the next two levels. The statistical shape model we use has approximately 18,000 points. As we are not interested in a perfect segmentation of the bone, but only in a good general alignment we subsample this model by a factor of 9 in order achieve a higher speed performance.

After alignment of the femur shape model, we can define the lowest femur point and the two condyles exactly. The joint plane separating femur and tibia and containing both menisci is based on the aligned shape model of the tibia because the menisci are embedded in the tibia plateau. We define three points on the tibia, which are deformed along with the model. These three points determine a plane defining the tibia plateau (see also Fig. 5 for an illustration of the points).

\section{RESULTS}

We evaluated the algorithm on $42 \mathrm{MR}$ images of knee joints. The images were acquired on a 3T Siemens TIM-TRIO scanner (Siemens, Erlangen, Germany). The participants were specifically recruited for this study, however acquisition was performed according to the standard imaging protocols. Despite the fact that apparently healthy volunteers were recruited, one volunteer had a ligament rupture (1 knee joint) and two volunteers exhibited bone edema (4 knee joints). IRB approval (Kantonale Ethikkommission Bern) was obtained and the participants gave written informed consent. One data set was obtained from the Toshiba data base, acquired on a 1.5T Toshiba MRT200SP6 scanner (Toshiba Medical Systems, Otawara, Japan). In terms of accuracy, the results of the method have been validated retrospectively using manual landmark definitions by an expert and checked by an orthopedic surgeon. The manual definitions were done on 3D volumetric MR images, which were acquired for each dataset together with the scout and diagnostic images. These high-quality images have a resolution of less than $1 \mathrm{~mm}$ in each direction (imaging parameters: $3 \mathrm{D}$ gradient echo sequence with $T_{\mathrm{R}}=11 \mathrm{~ms}$ and $T_{\mathrm{E}}=$ $4.92 \mathrm{~ms}$ on Siemens machines and with $T_{\mathrm{R}}=9.1 \mathrm{~ms}$ and $T_{\mathrm{E}}=3.5 \mathrm{~ms}$ on Toshiba machines). They were acquired for validation and visualization purposes only and were not used by the algorithm. Additionally, we also evaluated the robustness of the method in a qualitative way by judging if the result for the OBB and joint plane was "perfect" (manual definition would have been similar retrospectively), "acceptable" (manual definition would have been different retrospectively, but relevant region is fully covered and inclination is reasonable) or "bad" (relevant region is not fully covered or inclination is wrong). 
Results for the Definition of the Oriented Bounding Box

The scout images, on which the scan planning was performed, usually contained 3 slices in coronal, sagittal and axial view (see Fig. 2). The images had an intra-slice resolution of $0.5-0.7 \mathrm{~mm}$ and an inter-slice spacing of 9-10 $\mathrm{mm}$ (imaging parameters: $2 \mathrm{D}$ gradient echo sequence with $T_{\mathrm{R}}=7.7 \mathrm{~ms}$ and $T_{\mathrm{E}}=3.67 \mathrm{~ms}$ on the Siemens system and $2 \mathrm{D}$ gradient echo sequence with $T_{\mathrm{R}}=9.1 \mathrm{~ms}$ and $T_{\mathrm{E}}=3.5 \mathrm{~ms}$ on the Toshiba system). As it occurs in clinics, the images covered the knee joint only partially and were sometimes shifted with respect to the center of the knee joint, which further complicated the task.

The laterality of the knee present in the locator image was correctly identified by the algorithm in all cases. The resulting oriented bounding box is shown for two cases in Fig. 4. For better visualization it is depicted in three views on a $\mathrm{T} 2$-weighted high-resolution volumetric image of the patient knee and not on the original scout image. The first row of Fig. 4 shows a good alignment: the knee joint is fully covered and the box is aligned with the posterior condyles of the femur, while the covered area is not too large. On the other hand, the image in the second row shows one case where the alignment with the condyles is not satisfactory.

Judging the robustness, $50 \%$ of the OBB were considered to be perfectly aligned, $40 \%$ were acceptable and $10 \%$ were bad. In most cases, the non-perfect alignment was caused by unsatisfactory orientation of the angle with the condyles (see bottom row of Fig. 4 for an illustration). For the quantitative analysis, we manually defined six landmarks on the femur, which determine the location and maximum extent of the knee joint, as well as the condyle rotation angle. The
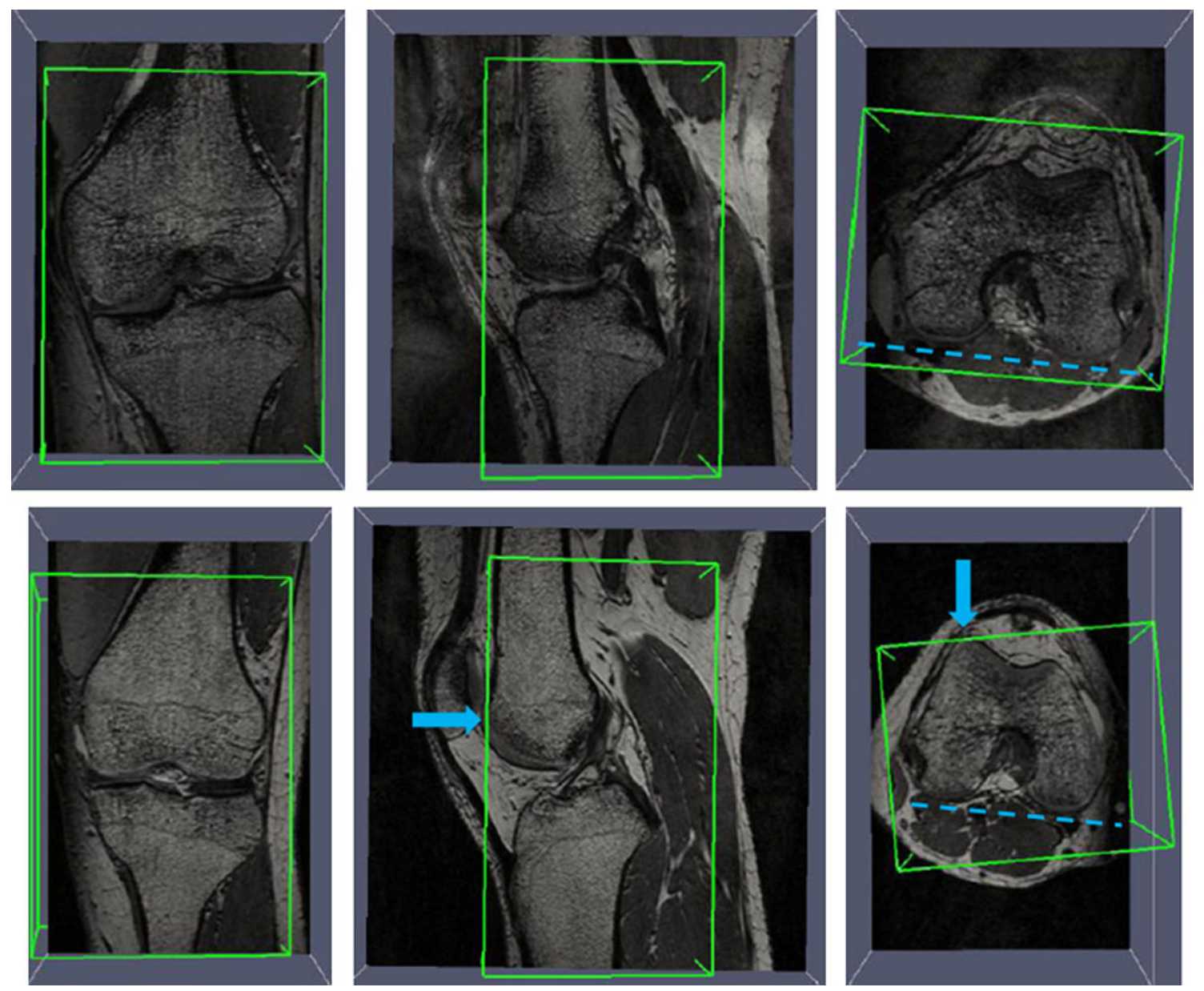

FIGURE 4. The oriented bounding box, which is found after Chamfer matching on the scout images (from left to right: coronal, sagittal and axial view). For better visual assessment, it is shown here on the 3D high-resolution images (3D gradient echo sequence, $T_{\mathrm{R}}=11 \mathrm{~ms}$ and $T_{\mathrm{E}}=4.92 \mathrm{~ms}$ ) and not on the scout images. Top row: one case with good results, the knee joint is completely covered, the bounding box is well aligned with the condyles and cover area is not too large. A line interconnecting both posterior condyles is shown in dashed blue to illustrate the good orientation of the alignment angle with the condyles. Bottom row: one case with bad results: the bounding box is not well aligned with the condyles (compare to the manually drawn dashed blue line, interconnecting both posterior condyles), the knee joint is not completely covered in the anterior part (blue arrows) and on the lateral side the covered area is too large. 
average accuracy of the landmarks defining the bounding box was $5.3 \mathrm{~mm}$ with a standard deviation of $4.3 \mathrm{~mm}$ and the average error of the condyle rotation angle was $6^{\circ}$ with a standard deviation of $5^{\circ}$. The individual accuracies for each point are shown in Table 1. The four cases which were judged to be "bad", were not considered for the quantitative analysis. In general, the cases in which the knee joint was covered only partially or shifted, were more frequent than the cases of failure, and they did not coincide with the cases where the algorithm returned bad results. The failures could neither be attributed to uncommon structures of the knee joint nor to the pathologic cases, but a general pattern which caused unsatisfactory results could not be identified.

Acquisition time for the scout image was $6 \mathrm{~s}$ per view, so $18 \mathrm{~s}$ in total. Computation time on a $2.3 \mathrm{GHz}$ quad-core processor with $3 \mathrm{~GB}$ of RAM was $18 \mathrm{~s}$ on average, which means also around $6 \mathrm{~s}$ per view.

TABLE 1. Accuracy of the automatic alignment of the oriented bounding box based on the scout images, compared to manual landmark definitions on the high-resolution volumetric images.

\begin{tabular}{lcc}
\hline & Mean & $\begin{array}{r}\text { Standard } \\
\text { deviation }\end{array}$ \\
\hline Anterior $(\mathrm{mm})$ & 5.6 & 4.5 \\
Posterior $(\mathrm{mm})$ & 6.7 & 4.6 \\
Lateral $(\mathrm{mm})$ & 4.4 & 2.9 \\
Medial $(\mathrm{mm})$ & 5.1 & 3.5 \\
Axial $(\mathrm{mm})$ & 4.5 & 5.7 \\
Angle $\left({ }^{\circ}\right)$ & 6.1 & 4.9 \\
Time $(\mathrm{s})$ & 17.5 & 2.2 \\
\hline
\end{tabular}

We show the mean error and its standard deviation for each corner separately, excluding the four cases where the method failed.

\section{Results for the Definition of the Joint Plane}

The diagnostic images for definition of the joint plane were acquired in sagittal direction covering the knee joint completely. They had an intra-slice resolution of $0.3-0.4 \mathrm{~mm}$ and an inter-slice spacing of 3.6-3.9 mm, with 25 slices on average (imaging parameters: 2D spin echo sequence with $T_{\mathrm{R}}=550 \mathrm{~ms}$ and $T_{\mathrm{E}}=16 \mathrm{~ms}$ on the Siemens system and 2D spin echo sequence with $T_{\mathrm{R}}=540 \mathrm{~ms}$ and $T_{\mathrm{E}}=15 \mathrm{~ms}$ on the Toshiba system).

The aligned models of femur and tibia after active shape modeling are shown in coronal, sagittal and axial view in Fig. 5 as an overlay on the T2-weighted high-resolution volumetric image of the patient knee (for better visualization not on the original sagittal diagnostic image). Both models are well aligned with the bones. The red crosses indicate the landmark points on the model used to determine both posterior condyles of the femur (two points) and the joint plane with both menisci on the tibia (three points).

In Fig. 6, the joint plane is depicted, which was automatically found from the three points defined on the tibia model for the same case as the one shown in Fig. 5. On the left, the plane is shown in coronal view and in the center in sagittal view. The right image shows the oblique plane itself in axial view. It is obvious that both menisci can be well seen on this automatically selected slice and it can also be seen that the position and inclination of the plane corresponds well to the patient anatomy.

Figure 7 shows a comparison of the manual definition of the joint plane on the left and the automatic definition of the joint plane in the center for one patient. It can be seen that for both cases, the menisci
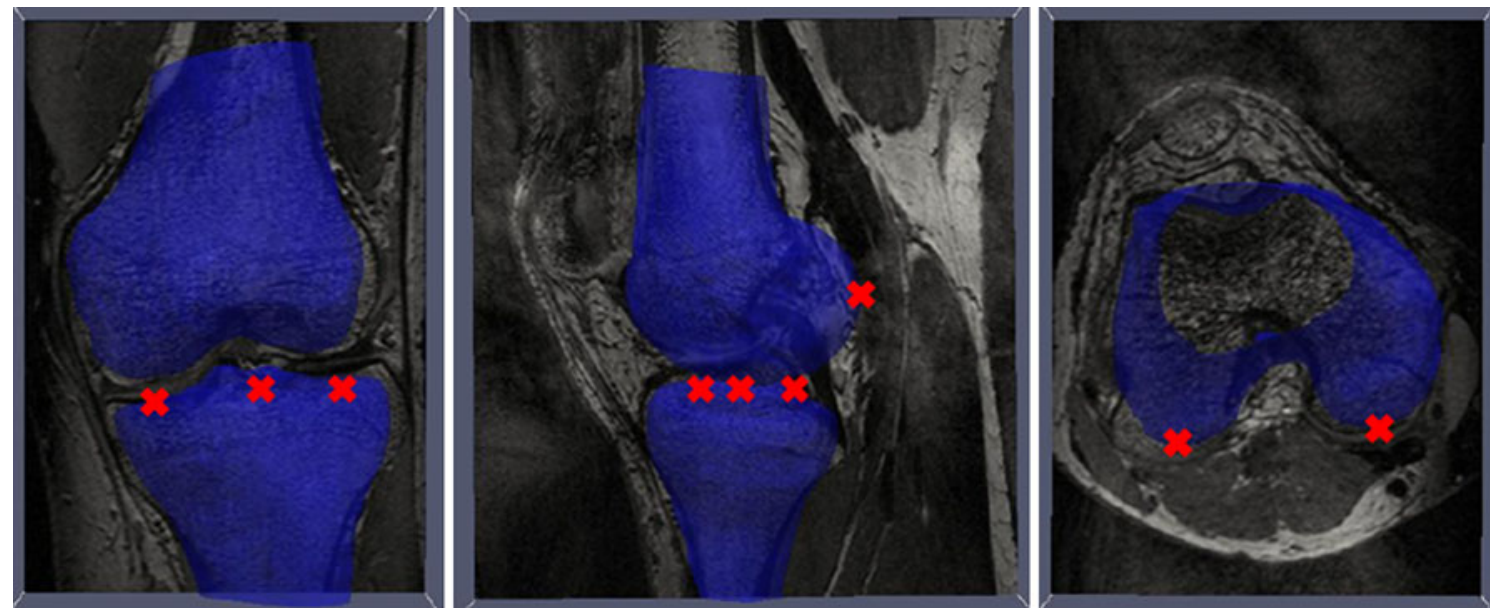

FIGURE 5. The shape model after alignment. For better visualization the result is shown on the high-resolution 3D image and not on the diagnostic image (from left to right: coronal, sagittal and axial view). Both femur and tibia models are well aligned with the structures of interest. The red crosses indicate the landmark points on the model, which are used to determine the joint plane ( 3 points on the tibia) and the posterior condyles (two points on the femur). 

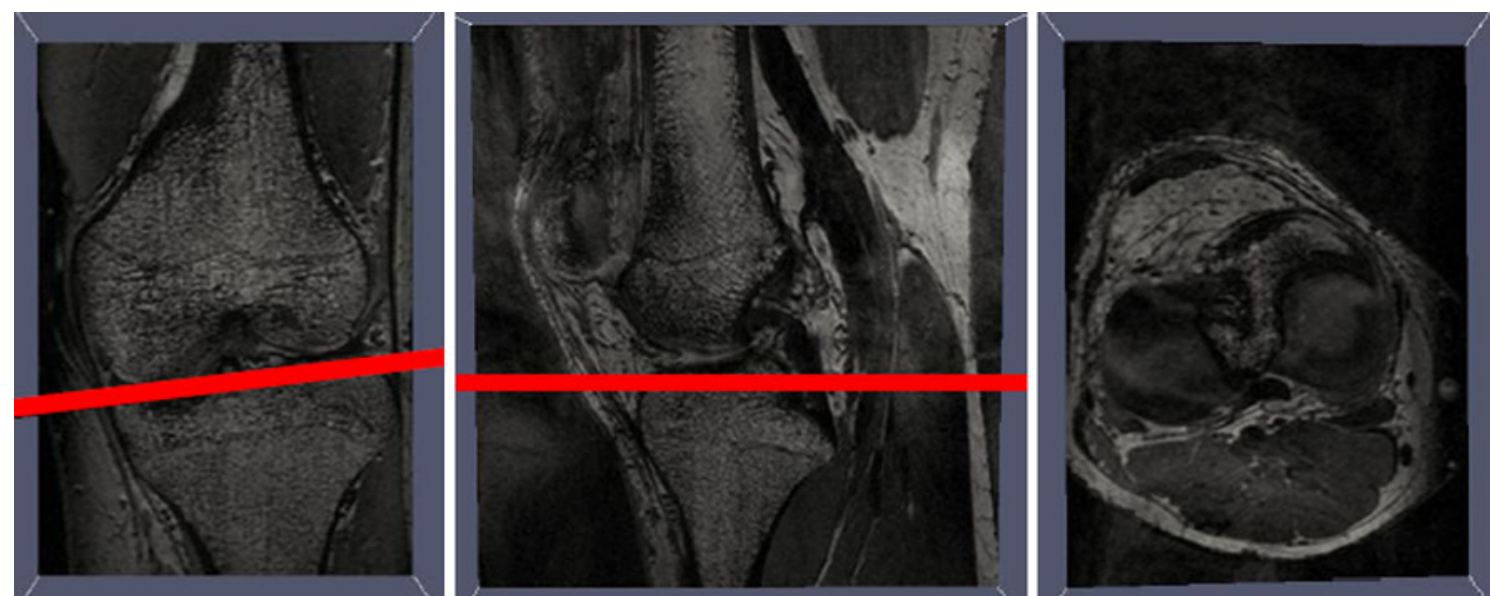

FIGURE 6. The joint plane, which was automatically found by the active shape model for the case depicted in Fig. 5. Left: joint plane (shown in red) in coronal view, center: joint plane (shown in red) in sagittal view, right: joint plane in axial view. The figure on the right shows that the oblique joint plane contains both menisci.
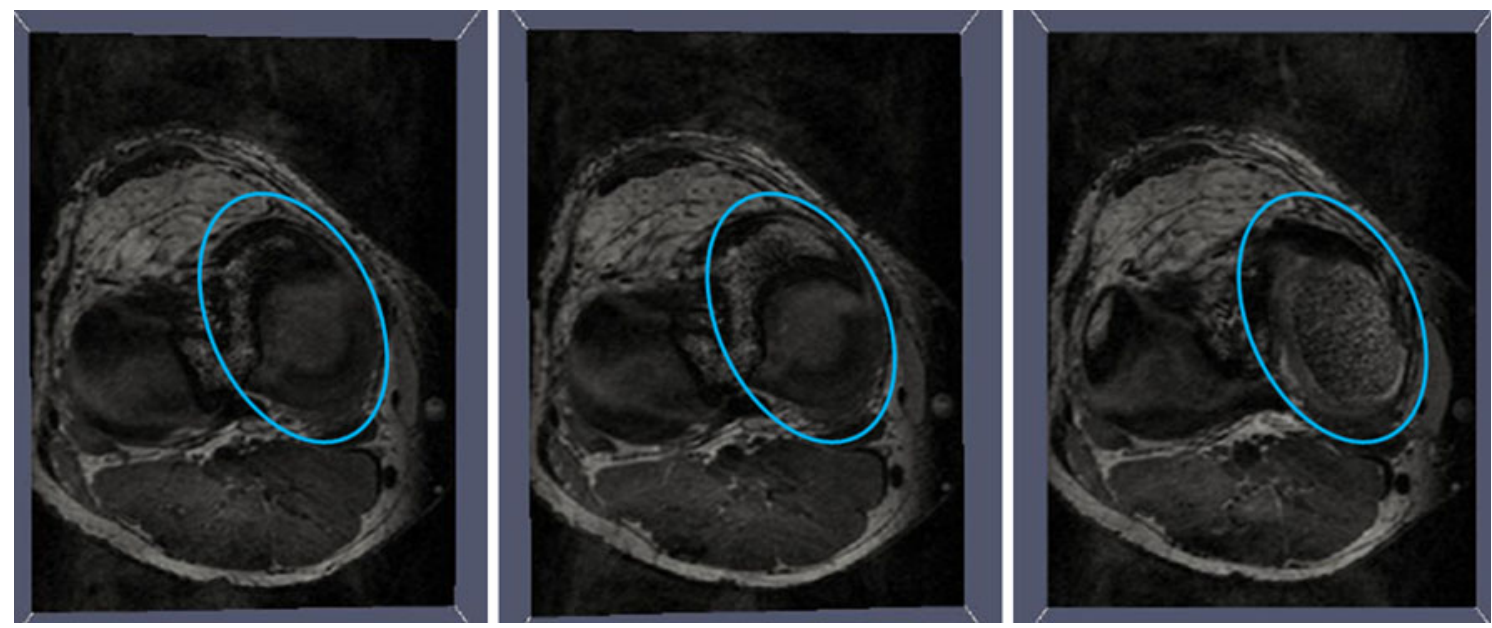

FIGURE 7. Comparison of the manual definition of the joint plane on the left and automatic definition of the joint plane in the center, shown on the same patient case as Figs. 5 and 6 . The right image shows the result if only the best slice in $z$-direction is chosen, without considering the inclination. It can be seen that in this case only one meniscus is partially covered, while the other is missing completely in this slice (circled area).

are present, whereas the manual definition covers a slightly larger part of cartilage thanks to a slightly better inclination of the plane in the volumetric image (circled area). Additionally in the picture on the right it is illustrated that it is not sufficient to choose the best axis-aligned slice in $z$-direction because without considering the inclination, only one meniscus is partially covered in the image, while the other is missing completely (circled area).

All knee images were included in the analysis of the joint plane location, including those for which the initialization by the OBB method was considered to be unsatisfactory. Judging qualitatively, in $81 \%$ of all cases the selected plane was evaluated to be perfect, in $14 \%$ it was acceptable and in $5 \%$ of all cases the method failed. Failure was caused by a shift of the plane position in $z$-direction (due to bad initialization by the OBB method), so that the plane showed bone instead of cartilage. Based on the high-resolution volumetric images, an expert defined the joint plane containing both menisci manually. The outcome of the algorithm for the plane origin and plane normal was compared to the result of the expert. The two cases, when the method failed were left out for the quantitative results. The average transversal deviation of the automatic joint plane definition from the manual definition was $1.2 \mathrm{~mm}$ with a standard deviation of $0.8 \mathrm{~mm}$. The average difference between the plane normals was $3.6^{\circ}$ with a standard deviation of $2.5^{\circ}$ (see also Table 2). It should be emphasized, that the clinician doing the manual definition found it very difficult and time-consuming to perform the manual annotation 
TABLE 2. Accuracy of the automatic definition of the joint plane based on the sagittal diagnostic images compared to the manual definition on the high-resolution volumetric images.

\begin{tabular}{lrc}
\hline & Mean & $\begin{array}{r}\text { Standard } \\
\text { deviation }\end{array}$ \\
\hline z-Direction $(\mathrm{mm})$ & 1.2 & 0.8 \\
Angle $\left(^{\circ}\right)$ & 3.6 & 2.5 \\
Time $(\mathrm{s})$ & 20.5 & 3.1 \\
\hline
\end{tabular}

We show the mean and the standard deviation over all datasets for the deviation in axial direction and the inclination of the plane, excluding the two failed cases.

in the volumetric images. Therefore, when comparing the outcome of the manual and automatic method retrospectively, in some cases the automatic method was considered to be even better. Computation time depended on the convergence speed of the method. On average, the joint plane was found within $21 \mathrm{~s}$.

\section{DISCUSSION AND CONCLUSION}

We presented a method to perform automatic scan planning for acquisition of diagnostic magnetic resonance images of the knee joint. This method was combined with a technique to automatically localize the joint plane containing both menisci from the diagnostic images. In contrast to other approaches suggested so far, the method at hand can work on the currently used 2D scout imaging protocols and does not require a new volumetric scout sequence, which has a longer acquisition time. The automatic joint plane localization can help the clinicians to analyze the diagnostic sequences in shorter time and in an optimized fashion such that diagnostic accuracy and reproducibility is improved. This is particularly important in serial examinations, e.g., to follow the development of degenerative processes, healing of tendons following surgery, cartilage repair in interventional studies, etc.

The approach was tested under challenging conditions with images from different machines and sites, as well as different patient groups. The accuracy and robustness proved to be sufficient in the majority of cases, although a further increase in robustness for the bounding box definition would be beneficial for even less need for human intervention. The method for scan planning is almost twice as accurate as the resolution of the scout images on which it operates $(5 \mathrm{~mm}$ average landmark error vs. 9-10 $\mathrm{mm}$ slice spacing) and it can find the borders of structures which are not present in the images at all. This can only be achieved thanks to the model-based approach, which is able to infer for example the tip of the condyles from the alignment of the whole model based on other parts of the femur. Computation time was sufficiently fast, so that the method can be used in a clinical scenario. As the images are processed in the same order as they are acquired, the previous acquisition can be analyzed while the next view is already being acquired. This reduces overall computation time, meaning the oriented bounding box, which defines the limits for the diagnostic acquisition, is available only $6 \mathrm{~s}$ after the acquisition of the scout images has finished. The accuracy of the bounding box detection on the scout images is in a similar range, although slightly lower compared to Bystrov et al. ${ }^{4}$ It is lower compared with Zhan et al. ${ }^{18}$ however both competing methods use a non-conventional 3D scout image, which contains much more information and they do not provide a delineation of the joint plane.

The method at hand has the potential to significantly speed-up the clinical workflow for acquisition of diagnostic knee MR images compared to the manual bounding box definition. The speed-up can in return lead to cost-savings in hospitals because more patients can be scheduled within the same time period and the technician is free to perform other tasks in the meantime. It has the potential to be easily incorporated into existing systems because it does not change the current acquisition protocol, it does not require any special computation hardware and is fast enough for clinical use.

\section{ELECTRONIC SUPPLEMENTARY MATERIAL}

The online version of this article (doi: 10.1007/s10439-012-0552-1) contains supplementary material, which is available to authorized users.

\section{ACKNOWLEDGMENTS}

We would like to thank Karin Zwygart for helping with data acquisition. We would also like to acknowledge Dr. Yasuo Sakurai for helpful discussions and data collection.

\section{REFERENCES}

\footnotetext{
${ }^{1}$ Blumenfeld, J., J. Carballido-Gamio, R. Krug, D. J. Blezek, I. Hancu, and S. Majumdar. Automatic prospective registration of high-resolution trabecular bone images of the tibia. Ann. Biomed. Eng. 35:1924-1931, 2007.

${ }^{2}$ Borgefors, G. Hierarchical chamfer matching: a parametric edge matching algorithm. IEEE Trans. Pattern Anal. Mach. Intell. 10:849-865, 1988.
} 
${ }^{3}$ Bou Sleiman, H., L. E. Ritacco, L. Aponte-Tinao, D. L. Muscolo, L.-P. Nolte, and M. Reyes. Allograft selection for transepiphyseal tumor resection around the knee using three-dimensional surface registration. Ann. Biomed. Eng., 2011. doi:10.1007/s10439-011-0282-9.

${ }^{4}$ Bystrov, D., V. Pekar, S. Young, S. P. M. Dries, H. S. Heese, and A. M. van Muiswinkel. Automated planning of MRI scans of knee joints. Proc. of SPIE 6509:65092Z65092Z-9, 2007.

${ }^{5}$ Canny, J. A computational approach to edge detection. IEEE Trans. Pattern Anal. Mach. Intell. PAMI-8:679-698, 1986.

${ }^{6}$ Cootes, T. F. Active shape models - their training and application. Comput. Vis. Image Underst. 61:38-59, 1995.

${ }^{7}$ Goldenstein, J., J. Schooler, J. C. Crane, E. Ozhinsky, J.-B. Pialat, J. Carballido-Gamio, and S. Majumdar. Prospective image registration for automated scan prescription of follow-up knee images in quantitative studies. Magn. Reson. Imaging 29:693-700, 2011.

${ }^{8}$ Heimann, T., and H. P. Meinzer. Statistical shape models for 3D medical image segmentation: a review. Med. Image Anal. 13:543-563, 2009.

${ }^{9}$ Ibanez, L., W. Schroeder, L. Ng, J. Cates, et al. The ITK Software Guide. Citeseer, 2003.

${ }^{10}$ Itti, L., L. Chang, and T. Ernst. Automatic scan prescription for brain MRI. Magn. Reson. Med. 45:486-494, 2001.

${ }^{11}$ Jackson, C. E., M. D. Robson, J. M. Francis, and J. A. Noble. Computerised planning of the acquisition of cardiac MR images. Comput. Med. Imaging Graph. 28:411-418, 2004.
${ }^{12}$ Jolly, M. P., C. V. Alvino, B. L. Odry, X. Deng, J. Zheng, M. Harder, and J. Guehring. Automatic femur segmentation and condyle line detection in 3D MR scans for alignment of high resolution MR, 2010. doi:10.1109/ISBI. 2010.5490142.

${ }^{13}$ Kitware. VTK User's Guide. Kitware Inc., 2010.

${ }^{14}$ Kozic, N., S. Weber, P. Büchler, C. Lutz, N. Reimers, M. A. González Ballester, and M. Reyes. Optimisation of orthopaedic implant design using statistical shape space analysis based on level sets. Med. Image Anal. 14:265-275, 2010.

${ }^{15}$ Lecouvet, F. E., J. Claus, P. Schmitz, V. Denolin, C. Bos, and B. C. Vande Berg. Clinical evaluation of automated scan prescription of knee MR images. JMRI 29:141-145, 2009.

${ }^{16}$ Lee, S.-L., P. Horkaew, A. Darzi, and G.-Z. Yang. Optimal scan planning with statistical shape modelling of the Levator Ani., 2003. doi:10.1007/978-3-540-39899-8 87.

${ }^{17}$ Ostlere, S. Imaging the knee. Imaging 19:249-268, 2007.

${ }^{18}$ Zhan, Y., M. Dewan, M. Harder, A. Krishnan, and $\mathrm{X}$. Zhou. Robust automatic knee $\mathrm{mr}$ slice positioning through redundant and hierarchical anatomy detection. IEEE Trans. Med. Imaging 1-14, 2011. doi:10.1109/TMI. 2011.2162634.

${ }^{19}$ Zhan, Y., M. Dewan, and X. S. Zhou. Auto-alignment of knee MR scout scans through redundant, adaptive and hierarchical anatomy detection. Inf. Process. Med. Imaging 22:111-122, 2011.

${ }^{20}$ Zitova, B., and J. Flusser. Image registration methods: a survey. Image Vis. Comput. 21:977-1000, 2003. 